\title{
The effect of cardiac angiography timing, contrast media dose, and preoperative renal function on acute renal failure after coronary artery bypass grafting
}

\author{
Benjamin Medalion, MD, ${ }^{\mathrm{a}}$ Hilit Cohen, MD, ${ }^{\mathrm{a}}$ Abid Assali, MD, ${ }^{\mathrm{b}}$ Hana Vaknin Assa, MD, ${ }^{\mathrm{b}}$ \\ Ariel Farkash, MD, ${ }^{\text {a }}$ Eitan Snir, MD, ${ }^{\mathrm{a}}$ Erez Sharoni, MD, ${ }^{\mathrm{a}}$ Philip Biderman, MD, ${ }^{\mathrm{a}}$ Gai Milo, MD, \\ Alexander Battler, MD, ${ }^{\mathrm{b}}$ Ran Kornowski, MD, ${ }^{\mathrm{b}}$ and Eyal Porat, $\mathrm{MD}^{\mathrm{a}}$
}

Objective: Our objective was to assess the effect of the timing of cardiac angiography, contrast media dose, and preoperative renal function on the prevalence of acute renal failure after cardiac surgery.

Methods: Data on 395 consecutive patients who underwent coronary artery bypass grafting were prospectively
collected. Creatinine clearance was estimated by the Cockcroft-Gault equation. Patients were divided into 3
groups according to the time between cardiac angiography and surgery (group $\mathrm{A}, \leq 1$ day; group $\mathrm{B},>1$
day and $\leq 5$ days; group $\mathrm{C},>5$ days). Patients who underwent a salvage operation or were receiving dialysis
before surgery were excluded. Acute renal failure was defined as $25 \%$ decrease from baseline of estimated
creatinine clearance and estimated creatinine clearance of $60 \mathrm{~mL} / \mathrm{min}$ or less on postoperative day 3 . Owing
to differences in preoperative characteristics between groups, propensity score analysis was used to adjust those
differences.

Results: Acute renal failure developed in $13.6 \%$ of patients. Hospital mortality was $3.3 \%$ and was higher in patients in whom acute renal failure developed $(22 \%)$ versus those in whom it did not $(0.3 \% ; P<.001)$. Multivariable analysis identified preoperative estimated creatinine clearance of $60 \mathrm{~mL} / \mathrm{min}$ or less (odds ratio [OR], 7.1), operation within 24 hours of catheterization $(\mathrm{OR}=3.7)$, use of more than $1.4 \mathrm{~mL} / \mathrm{kg}$ of contrast media $(\mathrm{OR}=$ $3.4)$, lower hemoglobin level $(\mathrm{OR}=1.3)$, older age $(\mathrm{OR}=1.1)$, and lower weight $(\mathrm{OR}=0.95)$ as independent predictors of postoperative acute renal failure. Analysis of interaction between contrast dose and time of surgery revealed that high contrast dose $(>1.4 \mathrm{~mL} / \mathrm{kg})$ predicted acute renal failure if surgery was performed up to 5 days after angiography.

Conclusions: Whenever possible, coronary bypass grafting should be delayed for at least 5 days in patients who received a high contrast dose, especially if they also have preoperative reduced renal function. (J Thorac Cardiovasc Surg 2010;139:1539-44)

Acute renal failure (ARF) has been shown to be associated with worse outcome after cardiac surgery. ${ }^{1-4}$ Contrast-induced nephropathy (CIN) is a common cause of new $\mathrm{ARF}^{5,6}$ and is related in part to the amount of contrast used. ${ }^{7}$ As such, the timing between angiography and surgery may be an important factor in the development of ARF. The purpose of this study was to identify the association between time of angiography, time of surgery, amount of contrast used, and postoperative ARF and its consequences.

\footnotetext{
From the Departments of Cardiothoracic Surgery, ${ }^{\mathrm{a}}$ Cardiology, ${ }^{\mathrm{b}}$ and Nephrology, ${ }^{\mathrm{c}}$ Rabin Medical Center, Beilinson Campus, Petach Tiqva, Israel. Affiliated with Sackler School of Medicine, Tel Aviv University, Tel Aviv, Israel.

Disclosures: None.

Received for publication Feb 19, 2009; revisions received Aug 23, 2009; accepted for publication Oct 10, 2009; available ahead of print Dec 7, 2009.

Address for reprints: Benjamin Medalion, MD, Department of Cardiothoracic Surgery, Rabin Medical Center, Beilinson Campus, Petach Tiqva, 49100, Israel (E-mail: benjamin2@clalit.org.il).

0022-5223/\$36.00

Copyright (C) 2010 by The American Association for Thoracic Surgery

doi:10.1016/j.jtcvs.2009.08.042
}

\section{METHODS \\ Study Population}

This is a retrospective study based on a local database in which data are prospectively collected. The database was approved by the hospital ethics committee for research purposes. The database includes preangiography, angiography, operative, and perioperative data. To have as homogeneous a group of patients and as complete and reliable data as possible, the study population included only patients having isolated first-time coronary artery bypass grafting $(\mathrm{CABG})$ who were catheterized and operated on in our facility with the use of cardiopulmonary bypass. Patients referred for surgery from other hospitals were excluded. Patients who required a salvage operation or were receiving dialysis before surgery were also excluded. Between January 2006 and July 2007, 395 patients fulfilled these criteria and served as the study population.

\section{Data Definitions and End Points}

We estimated creatinine clearance $(\mathrm{eClCr})$ for all patients before angiography and on postoperative day 3 by the Cockcroft-Gault formula, ${ }^{8}([140-$ age $\cdot$ body weight $[\mathrm{kg}] / \mathrm{serum}$ creatinine $[\mathrm{mg} / \mathrm{dL}] \cdot 72$ ) (multiply by 0.85 in women). Preangiography renal failure was defined as eClCr of $60 \mathrm{~mL} / \mathrm{min}$ or less. This is consistent with moderate renal dysfunction (level 3 of the National Kidney Foundation classification). ${ }^{9}$ The primary end point of the study was development of ARF after surgery, defined as absolute eClCr of $60 \mathrm{~mL} / \mathrm{min}$ or less on postoperative day 3 , in addition to $25 \%$ decrease in $\mathrm{eClCr}$ on postoperative day 3 relative to preangiography value, consistent 


$$
\begin{aligned}
& \text { Abbreviations and Acronyms } \\
& \begin{aligned}
\mathrm{ARF} & =\text { acute renal failure } \\
\mathrm{CABG} & =\text { coronary artery bypass grafting } \\
\mathrm{CIN} & =\text { contrast-induced nephropathy } \\
\mathrm{eClCr} & =\text { estimated creatinine clearance } \\
\mathrm{ICU} & =\text { intensive care unit } \\
\mathrm{OR} & =\text { odds ratio }
\end{aligned}
\end{aligned}
$$

with the definition of CIN.$^{10}$ Cutoff point for high contrast dose was defined as upper $25 \%$ values of contrast administered per kilogram. In this study, it was found to be $1.4 \mathrm{~mL} / \mathrm{kg}$. Secondary end points of the study were perioperative mortality defined as in-hospital mortality or 30-day mortality, prolonged intensive care unit (ICU) stay, and prolonged hospital stay (from operation to discharge).

\section{Angiographic Protocol}

Low-osmolarity contrast (Ultravist; Schering AG, Berlin, Germany) is used routinely in all patients. In patients with an eClCr of $60 \mathrm{~mL} / \mathrm{min}$ or less before angiography, known nephrotoxic medications are discontinued 24 hours before angiography. In addition, hydration with $1000 \mathrm{~mL}$ of normal saline solution is performed over several hours before the procedure. A dose of $1200 \mathrm{mg} \mathrm{N}$-acetylcysteine is administered every 12 hours, 1 day before angiography and 1 day after angiography (patients operated on the same day as angiography did not get the postangiography dose). This protocol is supported by current knowledge that the aforementioned measures reduce the prevalence of CIN. ${ }^{10}$

\section{Data Analysis}

We divided our study population into 3 groups on the basis of the time interval between angiography and surgery: group A, interval 1 day or less $(\mathrm{n}=153)$; group $\mathrm{B}$, interval more than 1 day and less than 5 days $(\mathrm{n}=$ $120)$; and group $C$, interval 5 days or more $(n=122)$. An interval of 5 days was selected to make sure that CIN had already peaked. An interval of 1 day was selected to isolate higher risk patients in whom same-day surgery was mandatory. We used the logistic EuroSCORE model to predict perioperative mortality for each patient ${ }^{11}$ and a CIN score, as suggested by Mehran and associates, ${ }^{7}$ to predict postcatheterization CIN. The higher the CIN score, the higher the probability that CIN would develop after catheterization. Preoperative and operative characteristics are displayed in Table 1. Several differences between groups are apparent. There is a higher rate of diabetes and hypertension in group $\mathrm{C}$, the creatinine level is higher, and $\mathrm{eClCr}$ is lower in group C. These factors affect CIN score, which is higher in this group. In contrast, more patients in group A had unstable angina and were operated on urgently, reflected by higher perioperative predicted mortality (EuroSCORE)

Adjustment for patient selection. To further adjust for patient selection and differences between patients, based on all preoperative variables, we used a saturated multinomial logistic regression model to calculate the probability that a patient would belong to 1 of the 3 groups. These propensity scores were later used in multivariable logistic regressions as a way for better adjustment of the preoperative differences. ${ }^{12,13}$

To compare between groups, we used 1-way analysis of variance with post hoc Bonferroni pairwise comparisons for normally distributed continuous variables and the Kruskal-Wallis test for abnormally distributed continuous variables. For univariate outcome analysis, we compared normally distributed continuous variables by the $t$ test and abnormally distributed continuous variables by the Mann-Whitney test. Categorical variables were compared br $\chi^{2}$ or Fisher's exact test, as appropriate. Several multivariable binary logistic regression models, with forward conditional variable selection, were developed to identify independent associations with outcome. Propensity score was forced into all models. Analysis of interaction between time to surgery, eClCr, and contrast dose was also performed to search for additive effects. Each model was performed once with preoperative variables only and once with both preoperative and intraoperative variables. We included all preoperative and intraoperative variables in Table 1 in the models except CIN and EuroSCORE. In addition, we used $\mathrm{eClCr}$ and contrast dose in both its continuous forms and then in separate models in its categorical forms for better clinical inference, inasmuch as eClCr calculation is based on age, sex, serum creatinine, and weight. When eClCr was studied, the other variables were omitted and vice versa to reduce colinearity and redundant information.

Reliability of the analysis. To ascertain the reliability of our findings, we used bootstrap aggregation (bagging) from automated analysis of 1000 bootstrap data sets. Variables appearing in $50 \%$ or more of the models were retained as risk factors. ${ }^{14}$

Analysis was performed with SPSS 14 for Windows (SPSS, Inc, Chicago, Ill).

We all had full access to the data and take responsibility for its integrity. All of us have read and agree to the manuscript as written.

\section{RESULTS \\ Primary End Point \\ Of 395 patients studied, 54 (13.6\%) had postoperative ARF.}

Univariate analysis. Univariate analysis identified several preoperative variables to be associated with the development of postoperative ARF (Table 2). Older age, female gender, advanced New York Heart Association class, diabetes, lower weight, lower hemoglobin level, and higher serum creatinine level were all found to have such an association. Of specific note are lower eClCr $(52 \%$ vs $11 \%$ of patients had preoperative eClCr $\leq 60 \mathrm{~mL} / \mathrm{min}$ in the presence and absence of postoperative ARF, respectively; $P<.001$ ), higher contrast dose $(43 \%$ vs $20 \%$ of patients received contrast dose $\geq 1.4 \mathrm{~mL} / \mathrm{kg}$ in the presence and absence of postoperative ARF, respectively; $P<.001$ ), and shorter interval between catheterization and surgery $(20 \%$ vs $12 \%$ vs $8 \%$ in groups $\mathrm{A}, \mathrm{B}$, and $\mathrm{C}$, respectively; $P=.02$ ), which increased postoperative ARF. Measured operative variables did not seem to be associated with ARF. CIN score was higher in those who in whom ARF developed (Table 2).

Multivariable analysis. Multivariable logistic regression identified several risk factors to be associated with development of postoperative ARF (Table 3). Preoperative eClCr of $60 \mathrm{~mL} / \mathrm{min}$ or less, contrast dose of $1.4 \mathrm{~mL} / \mathrm{kg}$ or more, operation within 1 day from angiography, and lower preoperative hemoglobin levels were found to be independently associated with postoperative ARF, with and without propensity adjustment. These variables also appeared more than $50 \%$ of the time as predictors in the 1000 automatic bootstrap analysis (see Methods) and, as such, appear to be reliable predictors. When analyzed with or without $\mathrm{eClCr}$ in the model, older age and lower weight also were risk factors for postoperative ARF development. Serum creatinine did not turn out to be an independent predictor for postoperative ARF. When the interaction of contrast dose of $1.4 \mathrm{~mL} /$ 
TABLE 1. Preoperative and operative characteristics

\begin{tabular}{|c|c|c|c|c|}
\hline & Group A $(n=153)$ & Group B $(n=120)$ & Group C $(n=122)$ & $P$ value \\
\hline \multicolumn{5}{|l|}{ Preoperative } \\
\hline Age (y) & $65.7 \pm 11$ & $66.2 \pm 10$ & $66 \pm 11$ & .89 \\
\hline Sex (female) & $34(22)$ & $27(22)$ & $27(18)$ & .99 \\
\hline NYHA class III or IV & $5(3)$ & $2(2)$ & $2(2)$ & .43 \\
\hline Unstable angina & $88(57)$ & $35(29)$ & $32(26)$ & .01 \\
\hline Urgent operation & $26(17)$ & $6(5)$ & $4(3)$ & $<.001$ \\
\hline Diabetes & $48(31)$ & $40(33)$ & $60(49)$ & .02 \\
\hline Hypertension & $94(61)$ & $85(71)$ & $94(77)$ & .02 \\
\hline Smoking history & $75(49)$ & $62(52)$ & $57(47)$ & .74 \\
\hline CVA & $14(9)$ & $12(10)$ & $11(9)$ & .96 \\
\hline PVD & $19(12)$ & $15(13)$ & $22(18)$ & .34 \\
\hline LVEF $(\%)$ & $53 \pm 9$ & $55 \pm 8$ & $54 \pm 10$ & .45 \\
\hline Weight $(\mathrm{kg})$ & $78 \pm 12$ & $77 \pm 12$ & $79 \pm 13$ & .47 \\
\hline $\mathrm{Hb}(\mathrm{g} / \mathrm{dL})$ & $13.5 \pm 1.7$ & $13.4 \pm 1.5$ & $13.3 \pm 1.8$ & .63 \\
\hline Creatinine $(\mathrm{mg} / \mathrm{dL})$ & $0.94 \pm 0.26$ & $1.01 \pm 0.4$ & $1.07 \pm 0.6$ & $.025 *$ \\
\hline $\mathrm{eClCr}(\mathrm{mL} / \mathrm{min})$ & $88 \pm 26$ & $82 \pm 22$ & $79 \pm 27$ & $.02 *$ \\
\hline $\mathrm{eClCr}(\leq 60 \mathrm{~mL} / \mathrm{min})$ & $20(13)$ & $18(15)$ & $26(21)$ & .17 \\
\hline Contrast used (mL/kg) & $1.1 \pm 0.7$ & $1.2 \pm 0.6$ & $1.1 \pm 0.5$ & .17 \\
\hline Contrast used $(\geq 1.4 \mathrm{~mL} / \mathrm{kg})$ & $39(25)$ & $30(25)$ & $23(19)$ & .38 \\
\hline CIN score & $3.3 \pm 3$ & $3.4 \pm 3$ & $4.2 \pm 3$ & $.05^{*}$ \\
\hline EuroSCORE $(\%)$ & $7 \pm 4.3$ & $5.4 \pm 4.4$ & $5.2 \pm 4.3$ & $<.001 \dagger$ \\
\hline \multicolumn{5}{|l|}{ Operative } \\
\hline No. of grafts & $3(1-6)$ & $3(1-6)$ & $3(1-6)$ & .72 \\
\hline $\mathrm{XCT}(\min )$ & $68 \pm 25$ & $61 \pm 21$ & $72 \pm 28$ & .26 \\
\hline CPBT (min) & $84 \pm 32$ & $78 \pm 28$ & $90 \pm 39$ & .4 \\
\hline
\end{tabular}

Data are presented as mean \pm standard deviation or number of cases (percent), except for number of grafts, given as median (range). NYHA, New York Heart Association; $C V A$, cerebrovascular accident; $P V D$, peripheral vascular disease; $L V E F$, left ventricular ejection fraction; $H b$, hemoglobin; $e C l C r$, estimated creatinine clearance; $C I N$, contrast-induced nephropathy; $X C T$, crossclamp time; $C P B T$, cardiopulmonary bypass time. * Group A versus $\mathrm{C}$; †group A versus $\mathrm{B}$ and $\mathrm{C}$.

$\mathrm{kg}$ or more with group was studied as a variable (contrast $\mathrm{x}$ group), being in group $\mathrm{C}$ (more than 5 days between angiography and surgery) was found to be protective in the presence of a contrast dose of $1.4 \mathrm{~mL} / \mathrm{kg}$ or more (relative to groups A and $\mathrm{B} ; P=.02$ ). Graphic demonstration of the probability for the development of postoperative ARF is presented by the $95 \%$ confidence interval bars in Figure 1, categorized by contrast dose. It can be appreciated that surgery performed after 5 days from angiography carries a similar low risk of ARF regardless of the amount of contrast used. This is in contrast to surgery performed up to 5 days from angiography, which carries a higher risk of ARF when high contrast dose is used. All models showed good fit and calibration with nonsignificant Hosmer and Lemeshow tests $(P>.4)$ and receiver operating curve $c$-statistics (area under the curves $>0.7 ; P<.05$ ).

\section{Secondary End Points}

Postoperative length of stay was longer in patients in whom ARF developed (Table 2). Prolonged ICU stay ( $\geq 48$ hours) was present in $25 \%$ versus $10 \%$ in those in whom ARF did and did not develop, respectively $(P=$ $.004)$. Prolonged hospital stay ( $\geq 10$ days) was present in $12 \%$ versus $5 \%$ in those in whom ARF did and did not de- velop, respectively $(P=.05)$. Overall perioperative mortality was $3.3 \%$. It was $0.3 \%$ when no ARF developed versus $22 \%$ when ARF developed $(P<.001)$.

\section{Discrimination Power}

Inasmuch as the current study is too small to allow an independent scoring system to be developed to predict ARF after CABG, we assessed the discrimination power of the CIN score to predict postoperative ARF. We also modeled the time interval variable with CIN score in a multivariable logistic regression and assessed its ability to predict ARF. The predicted probability to develop ARF calculated from the multivariable analysis, the CIN score, and the predicted probability from the CIN and time interval model were used to generate receiver operating curves. Prediction from the multivariable model showed the best discrimination with area under the curve ( $c$-statistics) of 0.88 . It was 0.84 for the combined CIN score and time interval, and it was 0.82 for the CIN score alone.

\section{DISCUSSION}

ARF remains a major complication of CABG that is strongly associated with worse outcome. ${ }^{1-4}$ Indeed, in this study, patients in whom ARF developed had higher 
TABLE 2. Preoperative, operative, and postoperative univariate analysis of ARF

\begin{tabular}{|c|c|c|c|}
\hline & $\begin{array}{c}\text { No ARF } \\
(n=341)\end{array}$ & $\begin{array}{c}\text { ARF } \\
(\mathbf{n}=\mathbf{5 4}) \\
\end{array}$ & $\begin{array}{c}P \\
\text { value } \\
\end{array}$ \\
\hline \multicolumn{4}{|l|}{ Preoperative } \\
\hline Age (y) & $65 \pm 10$ & $74 \pm 11$ & $<.001$ \\
\hline Sex (female) & $70(21)$ & $18(33)$ & .04 \\
\hline NYHA class III or IV & $5(2)$ & $4(7)$ & .05 \\
\hline Unstable angina & $133(39)$ & $22(41)$ & .6 \\
\hline Urgent operation & $30(9)$ & $6(11)$ & .6 \\
\hline Diabetes & $121(35)$ & $28(52)$ & .004 \\
\hline Hypertension & $232(68)$ & $41(76)$ & .25 \\
\hline Smoking history & $171(50)$ & $23(43)$ & .3 \\
\hline CVA & $31(9)$ & $6(11)$ & .64 \\
\hline PVD & $51(15)$ & $5(9)$ & .3 \\
\hline $\operatorname{LVEF}(\%)$ & $54 \pm 9$ & $53 \pm 10$ & .27 \\
\hline Weight (kg) & $79 \pm 12$ & $73 \pm 13$ & $<.001$ \\
\hline $\mathrm{Hb}(\mathrm{g} / \mathrm{dL})$ & $13.6 \pm 1.6$ & $12.3 \pm 1.8$ & $<.001$ \\
\hline Creatinine $(\mathrm{mg} / \mathrm{dL})$ & $0.98 \pm 0.4$ & $1.1 \pm 0.4$ & $<.001$ \\
\hline $\mathrm{eClCr}(\mathrm{mL} / \mathrm{min})$ & $86 \pm 25$ & $67 \pm 21$ & $<.001$ \\
\hline $\mathrm{eClCr}(\leq 60 \mathrm{~mL} / \mathrm{min})$ & $36(11)$ & $28(52)$ & $<.001$ \\
\hline Contrast used (mL/kg) & $1.1 \pm 0.5$ & $1.3 \pm 0.8$ & .03 \\
\hline Contrast used $(\geq 1.4 \mathrm{~mL} / \mathrm{kg})$ & $69(20)$ & $23(43)$ & $<.001$ \\
\hline Time to surgery & & & .02 \\
\hline$\leq 1$ day $($ group $\mathrm{A})$ & $123(80)$ & $30(20)$ & \\
\hline 1-5 days (group B) & $106(88)$ & $14(12)$ & \\
\hline$\geq 5$ days (group $\mathrm{C}$ ) & $112(92)$ & $10(8)$ & \\
\hline CIN score & $3 \pm 3$ & $7 \pm 4$ & $<.001$ \\
\hline EuroSCORE $(\%)$ & $5 \pm 3$ & $7.5 \pm 4$ & $<.001$ \\
\hline \multicolumn{4}{|l|}{ Operative } \\
\hline No. of grafts & $3(1-6)$ & $3(1-6)$ & .7 \\
\hline XCT (min) & $67 \pm 26$ & $66 \pm 22$ & .87 \\
\hline CPBT (min) & $85 \pm 34$ & $80 \pm 24$ & .55 \\
\hline \multicolumn{4}{|l|}{ Postoperative } \\
\hline eClCr on day $3(\mathrm{~mL} / \mathrm{min})$ & $87 \pm 32$ & $50 \pm 21$ & $<.001$ \\
\hline ICU stay (h) & $35 \pm 69$ & $55 \pm 112$ & .003 \\
\hline Prolonged ICU stay $(>48 \mathrm{~h})$ & $32(10)$ & $12(25)$ & .004 \\
\hline Hospital stay (d) & $5.8 \pm 3.7$ & $7.2 \pm 4.6$ & .03 \\
\hline Prolonged hospital stay $(>10 \mathrm{~d})$ & $16(5)$ & $6(12)$ & .05 \\
\hline Perioperative mortality & $1(0.3)$ & $12(22)$ & $<.001$ \\
\hline
\end{tabular}

Data are presented as mean \pm standard deviation or number of cases (percent), except for number of grafts, given as median (range). $A R F$, Acute renal failure; $N Y H A$, New York Heart Association; $C V A$, cerebrovascular accident; $P V D$, peripheral vascular disease; $L V E F$, left ventricular ejection fraction; $\mathrm{Hb}$, hemoglobin; $e C l C r$, estimated creatinine clearance; $C I N$, contrast-induced nephropathy; $X C T$, crossclamp time; $C P B T$, cardiopulmonary bypass time.

perioperative mortality and longer ICU and hospital lengths of stay. Several preoperative and operative risk factors for development of ARF after cardiac surgery were identified; among them were older age, decreased preoperative eClCr, and reduced left ventricular function, to go along with isolated $\mathrm{CABG}$, previous $\mathrm{CABG}$, diabetes mellitus, congestive heart failure, urgent operation, and cardiopulmonary bypass time. ${ }^{1-4,15-17}$ Multiple mechanisms contribute to the renal damage, including nonpulsatile flow, embolization, trauma to the blood constituents, hypothermia, and activation of known inflammatory pathways. ${ }^{18}$
TABLE 3. Independent risk factors associated with ARF

\begin{tabular}{llccc}
\hline & OR & $\mathbf{9 5} \%$ CI & P value & Reliability (\%) \\
\hline eClCr $(\leq 60 \mathrm{~mL} / \mathrm{min})$ & 7.14 & $3-16$ & $<.001$ & 95 \\
Contrast used $(\geq 1.4 \mathrm{~mL} / \mathrm{kg})$ & 3.4 & $1.5-7.7$ & .004 & 70 \\
$\mathrm{Group} \mathrm{A}(\leq 1 \mathrm{~d})$ & 3.7 & $1.4-8.3$ & .07 & 73 \\
$\mathrm{Hb}(\mathrm{g} / \mathrm{dL})$ & 1.3 & $1.07-1.72$ & .01 & 75 \\
$\mathrm{Age}(\mathrm{y})$ & 1.1 & $1.06-1.16$ & $<.001$ & 90 \\
Weight $(\mathrm{kg})$ & 0.95 & $0.92-0.99$ & .005 & 64 \\
\hline ARF, Acute renal failure; OR, odds ratio; $C I$, confidence interval; reliability, percent of \\
1000 bootstrap analyses; eClCr, estimated creatinine clearance; $\mathrm{Hb}$, hemoglobin. \\
Odds ratios for Hb, age, and weight represent unit change, for eClCr $\leq 60$ and contrast \\
used $\geq 1.4$ represent presence of the variable relative to its absence, and for groups A \\
and B represent comparison to the average effect of group C.
\end{tabular}

Most studies have ignored CIN, a common cause of postangiography ARF. CIN develops in up to $10 \%$ of patients with normal renal function and may develop in up to $25 \%$ of patients with pre-existing renal impairment. ${ }^{10}$ Baseline renal impairment, diabetes mellitus, congestive heart failure, and higher doses of contrast media increase the risk of CIN. ${ }^{7,10,19}$ In the presence of a reduced nephron mass, the remaining nephrons are vulnerable to injury. Iodinated contrast, after causing a brief (minutes) period of vasodilation, causes sustained (hours to days) intrarenal vasoconstriction and ischemic injury. The ischemic injury sets off a cascade of events largely driven by oxidative injury, causing death of renal tubular cells. If a sufficient mass of nephron units are affected, then a recognizable rise in serum creatinine will occur. ${ }^{20}$ Several measures have been suggested to minimize CIN. ${ }^{6,10,19}$ Low-osmolarity contrast, hydration, and cessation of nephrotoxic medications are suggested

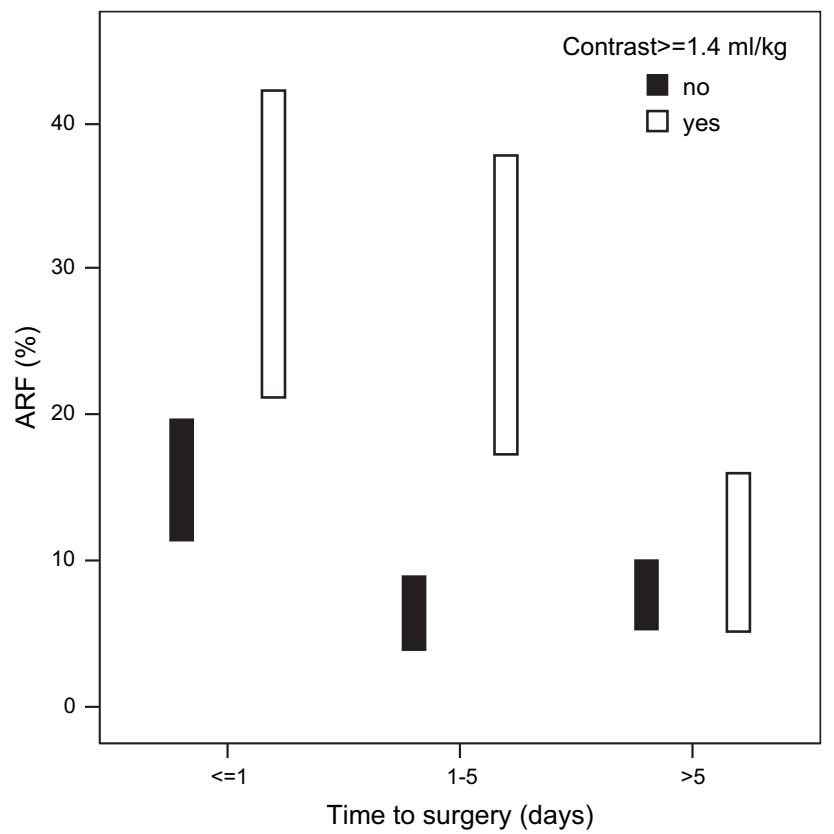

FIGURE 1. Predicted probability for the development of postoperative acute renal failure $(A R F)$ according to interval between angiography and surgery and contrast dose used. Bars represent $95 \%$ confidence interval. 
measures that are also part of our protocol in high-risk patients (see Methods). Inasmuch as CIN is usually a time-limited phenomenon, with an effect that peaks after 3 to 5 days from contrast administration, and inasmuch as contrast dose is an important factor in development of CIN, surgery soon after angiography may impose a second insult to the kidneys and induce ARF. High contrast dose was an independent predictor of postoperative ARF in this study, despite optimal protocol adherence to minimize CIN. However, its effect was mainly during the first 5 days after angiography, as described in the multivariable analysis by the interaction of contrast dose with time of surgery and graphically demonstrated in Figure 1. The association of high contrast dose and operation up to 5 days after angiography had an additive effect on the prevalence of ARF. Several reasons why patients received high dose of contrast media were identified. Ventriculograms were rarely performed in our institute, especially in patients with renal dysfunction, and are probably not the cause of high contrast dose. Contrast volume was determined by the number of injections and projections required for good quality of image and the need for delineation of the anatomy per request of the surgeon.

Other independent risk factors identified were older age, lower hemoglobin level, lower weight, and moderate preoperative renal failure. Lower hemoglobin level requires special attention. If it is truly an independent risk factor, then measures to increase hemoglobin preoperatively may prove to be beneficial. However, if lower hemoglobin is just a covariate to renal dysfunction, despite extensive statistical analysis, such measures are probably unnecessary. Future studies may clarify this issue.

Del Duca and associates ${ }^{16}$ found that surgery within 5 days of angiography was independently associated with postoperative ARF and mortality. Their study included several types of operations, including valve surgery with or without CABG and other operations that were already identified as risk factors for development of postoperative ARF. ${ }^{21}$ Moreover, these more complex operations increase cardiopulmonary bypass time, which by itself increases the risk for $\mathrm{ARF}^{3,15}$ In addition, inasmuch as contrast dose was not collected, and whether patients had any measure to reduce CIN is unclear, the association with these important factors could not be studied.

Ranucci and associates ${ }^{17}$ identified surgery within 1 day of angiography as a risk factor for postoperative ARF. They also studied mixed operations including reoperations that may represent more complicated cases in which ARF is likely to develop owing to multiple comorbidities. ${ }^{3}$ In contrast to our study, although suggested in univariate analysis, they failed to associate contrast dose with ARF in multivariable analysis. One explanation is that they used median contrast dose as their categorical cutoff point, which may not be high enough. We used the upper quartile of contrast dose as our categorical cutoff point and could show such an associ- ation. A second, and maybe more important, explanation relates to time of surgery. Inasmuch as CIN peaks after 3 to 5 days, CIN and ARF were more likely to develop in some patients who were operated on more than 1 day after angiography. By dividing our patient population into 3 groups we could show the CIN effect up to 5 days after angiography. They also used peak creatinine as their end point measure for development of ARF. This may further bias the results by capturing complicated cases in which ARF developed as part of a broader multiorgan failure, not directly related to time after angiography. Brown and associates, ${ }^{22}$ studied same-day angiography and elective valve surgery and concluded that in properly selected patients this is a safe option that carries little impact on postoperative ARF. They had no comparison group, they did not collect contrast dose, and more important, they excluded patients with significant renal dysfunction (serum creatinine $>1.8 \mathrm{mg} / \mathrm{dL}$ ). Indeed, even in our study, elective patients who had near normal preoperative renal function and received low contrast dose could undergo surgery safely immediately after angiography.

Owing to the worse outcome associated with ARF after CABG, a scoring system to predict its development will be important for both patient selection and risk stratification. This study is too small to develop and validate such a score, but we could show that even the angiographic CIN score can reasonably predict ARF in the surgical population. The addition of time from angiography to surgery can further refine the prediction and may serve as a starting point of surgical score calculation in a future larger study.

The main study limitation is its retrospective nature. To reduce the effect of this limitation, we have applied several actions. We excluded patients who had conditions that may have increased the risk for ARF. We excluded those who were receiving preoperative dialysis, those whose operations were done on a truly emergency basis, and those who had other operations in addition to CABG. We understood that despite exclusion of emergency cases, some patients who were operated on within 24 hours of angiography were higher risk patients, in whom same-day surgery was unavoidable, and may have been in worse preoperative condition and more prone to the development of ARF. Despite this, we included all those patients operated on within 24 hours in 1 group (group A). We also used a propensity scoring technique to correct for selection bias and performed bootstrap analysis to investigate how reliable the risk factors were that we identified.

It is important to emphasize that low-osmolarity contrast media (Ultravist) was used in all patients in this study. A higher rate of ARF is anticipated with the use of high-osmolarity contrast agents. Patients who had same-day surgery did not get the 12-hour postangiography $N$-acetylcysteine. After the results of this study, we continue to use $N$-acetylcysteine administration for 1 day after surgery. On the basis of this study, we can suggest that unless emergency CABG 
is absolutely indicated, surgery should be delayed for more than 5 days after angiography in patients who receive a high contrast dose, especially if they also have preoperative reduced renal function. If a patient is anticipated to require early operation, an attempt to minimize contrast dose is crucial, especially in the presence of reduced renal function. In addition, all measures should be taken to reduce ARF by using low-osmolarity contrast agent, with the lowest possible volume, good hydration, and probably $\mathrm{N}$-acetylcysteine administration.

\section{References}

1. Chertow GM, Levy EM, Hammermeister KE, Grover F, Daley J. Independent association between acute renal failure and mortality following cardiac surgery. Am J Med. 1998;104:343-8.

2. Lassnigg A, Schmidlin D, Mouhieddine M, Bachmann LM, Druml W, Bauer P, et al. Minimal changes of serum creatinine predict prognosis in patients after cardiothoracic surgery: a prospective cohort study. J Am Soc Nephrol. 2004;15: 1597-605.

3. Mangano CM, Diamondstone LS, Ramsay JG, Aggarwal A, Herskowitz A, Mangano DT. Renal dysfunction after myocardial revascularization: risk factors, adverse outcomes, and hospital resource utilization. The Multicenter Study of Perioperative Ischemia Research Group. Ann Intern Med. 1998;128:194-203.

4. Zanardo G, Michielon P, Paccagnella A, Rosi P, Calo M, Salandin V, et al. Acute renal failure in the patient undergoing cardiac operation: prevalence, mortality rate, and main risk factors. J Thorac Cardiovasc Surg. 1994;107:1489-95.

5. McCullough PA, Wolyn R, Rocher LL, Levin RN, O'Neill WW. Acute renal failure after coronary intervention: incidence, risk factors, and relationship to mortality. Am J Med. 1997;103:368-75.

6. Morcos SK. Contrast media-induced nephrotoxicity—questions and answers. $\mathrm{Br}$ J Radiol. 1998;71:357-65.

7. Mehran R, Aymong ED, Nikolsky E, Lasic Z, Iakovou I, Fahy M, et al. A simple risk score for prediction of contrast-induced nephropathy after percutaneous coronary intervention: development and initial validation. J Am Coll Cardiol. 2004;44:1393-9.

8. Cockcroft DW, Gault MH. Prediction of creatinine clearance from serum creatinine. Nephron. 1976;16:31-41.

9. K/DOQI clinical practice guidelines for chronic kidney disease: evaluation, classification, and stratification. Am J Kidney Dis. 2002;39:S1-266.

10. Kelly AM, Dwamena B, Cronin P, Bernstein SJ, Carlos RC. Meta-analysis: effectiveness of drugs for preventing contrast-induced nephropathy. Ann Intern Med. 2008;148:284-94.

11. Roques F, Michel P, Goldstone AR, Nashef SA. The logistic EuroSCORE. Eur Heart J. 2003;24:881-2.

12. Blackstone EH. Comparing apples and oranges. J Thorac Cardiovasc Surg. 2002; 123:8-15.

13. Rubin DB. Estimating causal effects from large data sets using propensity scores. Ann Intern Med. 1997;127:757-63.

14. Blackstone EH. Breaking down barriers: helpful breakthrough statistical methods you need to understand better. J Thorac Cardiovasc Surg. 2001;122:430-9.

15. Conlon PJ, Stafford-Smith M, White WD, Newman MF, King S, Winn MP, et al. Acute renal failure following cardiac surgery. Nephrol Dial Transplant. 1999;14: 1158-62.

16. Del Duca D, Iqbal S, Rahme E, Goldberg P, de Varennes B. Renal failure after cardiac surgery: timing of cardiac catheterization and other perioperative risk factors. Ann Thorac Surg. 2007;84:1264-71.

17. Ranucci M, Ballotta A, Kunkl A, De Benedetti D, Kandil H, Conti D, et al. Influence of the timing of cardiac catheterization and the amount of contrast media on acute renal failure after cardiac surgery. Am J Cardiol. 2008;101:1112-8.

18. Khalpey ZI, Ganim RB, Rawn JD. Postoperative care of cardiac surgery patients. In: Cohn LH, ed. Cardiac surgery in the adult. New York: McGraw-Hill; 2008: 465-86.

19. Barrett BJ. Contrast nephrotoxicity. J Am Soc Nephrol. 1994;5:125-37.

20. McCullough PA. Contrast-induced acute kidney injury. J Am Coll Cardiol. 2008; 51:1419-28.

21. Thakar CV, Arrigain S, Worley S, Yared JP, Paganini EP. A clinical score to predict acute renal failure after cardiac surgery. J Am Soc Nephrol. 2005;16:162-8.

22. Brown ML, Holmes DR, Tajik AJ, Sarano ME, Schaff HV. Safety of same-day coronary angiography in patients undergoing elective valvular heart surgery. Mayo Clin Proc. 2007;82:572-4. 